$62^{\text {ème }}$ Congrès de la SFCO, 02017 (2014)

DOI: $10.1051 /$ sfco/20146202017

(C) Owned by the authors, published by EDP Sciences, 2014

\title{
COMMUNICATION
}

\section{Métastase de lymphome lymphoblastique de type T au niveau de la cavité orale.}

\author{
Montaner S, Bahi-Gross S, Jung S, Gros Cl, Feki A
}

UF de Chirurgie Buccale-Implantologie. Pôle de médecine et chirurgie bucco-dentaires. Hôpital Civil, CHRU de Strasbourg, 1 place de l'hôpital, 67000 Strasbourg, FRANCE

Les lymphomes non hodgkiniens (LNH) forment un groupe hétérogène de maladies définies par une prolifération anormale de cellules lymphoïdes de lignées $B$ ( $85 \%$ des cas) ou plus rarement $\mathrm{T}$ ( $15 \%$ des cas). Le lymphome lymphoblastique $\mathrm{T}$ de l'adulte est une forme rare et agressive de lymphome non hodgkinien ( $2 \%$ des $\mathrm{LNH}$ ) survenant principalement chez l'adulte jeune et qui se manifeste généralement par une atteinte médullaire plus ou moins marquée (Monnereau et al. 2013).

Un patient de 62 ans est adressé par son chirurgien-dentiste traitant pour une tuméfaction maxillaire postérieure gauche en rapport avec une 28 en désinclusion évoluant depuis plus de 3 semaines. Dans ses antécédents, le patient rappelle la découverte d'une tumeur médiastinale réséquée 10 mois plus tôt et diagnostiquée comme un thymome.

L'examen clinique exobuccal est peu évocateur ; la palpation des aires ganglionnaires montre une adénopathie sous angulomaxillaire gauche douloureuse, dure à la palpation et mobile par rapport aux plans profonds. Au niveau endobuccal, l'examen révèle une lésion hypertrophique de plus de $2 \mathrm{~cm}$ recouverte d'une muqueuse érythémateuse et ulcérée. Les contours sont bien limités, la consistance est molle avec absence de fluctuation. Le sondage parodontal montre une poche de $7 \mathrm{~mm}$ en palatin et en mésial de la 28.

Cette tuméfaction initialement diagnostiquée comme un accident d'évolution de 28 a été traitée par son praticien par antibiothérapie à large spectre pendant 2 semaines associée à des manœuvres de drainage local qui se sont avérées infructueuses.
L'orthopantomogramme montre une 28 en désinclusion associée à une perte osseuse mésiale. L'examen par $\mathrm{CBCT}$ révèle une infiltration tissulaire au contact du maxillaire gauche avec une ostéolyse du fond du sinus maxillaire associé à un épaississement muqueux.

L'examen anatomopathologique réalisée sur un fragment de $1.4 \times 1 \mathrm{~cm}$ conclue à un lymphome lymphoblastique de type $\mathrm{T}$.

Le scanner cervico-thoraco-abdomino-pelvien injecté confirme l'ostéolyse focale au fond du sinus maxillaire gauche, et révèle des adénopathies cervicales gauches, des ganglions médiastinaux et une hépato- splénomégalie.

Devant ce tableau clinique évocateur et compte tenu des antécédents de thymome révélé lors de la consultation, les lames de la tumeur médiastinale ont été réexaminées. Il est apparu alors vraisemblable que le thymome épithélial diagnostiqué 10 mois plus tôt était un lymphome lymphoblastique T. C'est donc la « métastase lymphoblastique » orale qui a permis de remettre en cause le diagnostic initial et adapter le traitement.

Après un TEP Scan et un bilan d'extension montrant des localisations sous cutanées multiples et osseuses disseminées, une chimiothérapie suivant le protocole Graspall a été initiée.

Aucune manifestation orale de ce type de lymphome n'a été à ce jour répertoriée dans la littérature.

MONTANER Sylvain

montanersylvain@live.fr

This is an Open Access article distributed under the terms of the Creative Commons Attribution License 4.0, which permits unrestricted use, distribution, and reproduction in any medium, provided the original work is properly cited. 